\title{
A Strain-rate Dependant Micromechanical Finite Element Model for High-velocity Impacts on Laminated Composite Plates
}

\author{
Dimitris K. Siorikis ${ }^{1,}{ }^{*}$, Christos $V$. Nastos ${ }^{1}$, Dimitris A. Saravanos ${ }^{l}$ and Esteban Martino \\ Gonzalez $^{2}$ \\ ${ }^{1}$ Department of Mechanical Engineering and Aeronautics, Rion-Patras, Greece \\ ${ }^{2}$ Airbus, Madrid, Spain
}

\begin{abstract}
A novel multi-scale numerical model for the simulation of highvelocity impacts on laminated composite plates is developed, which encompasses a micromechanics module for the accurate assessment of damage initiation and growth in the individual composite micromechanical constituents and for the efficient inclusion of strain rate effects into the analysis. A series of woven carbon/epoxy plate specimens impacted by steel ball impactors of high velocities and energies reaching and exceeding the ballistic limit $(\mathrm{m}=110 \mathrm{~g}, \mathrm{v}=60-100 \mathrm{~m} / \mathrm{s}, \mathrm{E}=200-500 \mathrm{~J})$ are also investigated. Ultimately, key impact simulation results, such as the ballistic limit and induced impact damage, are correlated with representative experimental results, demonstrating the validity of the proposed impact model.
\end{abstract}

\section{Introduction}

Fiber-reinforced polymer matrix composites are increasingly applied in structural aerospace components due to their excellent mechanical properties. Despite the many attractive characteristics, laminated composite materials are susceptible to foreign object impact loading, such as impacts of small hail stones, engine blade fragments, bird strikes, runway debris, which may result in unpredictable complex damage and severely decrease the structural stiffness, stability, and load-carrying capacity.

In general, impacts of composite structures are highly complicated structural dynamic phenomena involving complex nonlinear interactions between the impactor and the target structure. Yet, the analysis of high-velocity impacts is further complicated by the development of extensive mixed-mode damage in the composite laminate and the nonnegligible strain-rate effects on the constitutive material behaviour [1]. Motivated by the need of developing a modelling tool for the simulation of counter rotating open rotor (CROR) blade impacts, and also by the shortage of impact simulation methods in terms of strain rate treatment $[2,3]$, the current work presents the development and validation of a multi-scale finite element model, which incorporates a rate-dependent constitutive model and a micromechanics model.

The multi-scale finite element model includes a micromechanics analysis scale based on the composite material model of Chamis [4]. The inclusion of micromechanics enables the synthesis of composite properties using constituent material models and input properties at the fiber/matrix level. Consequently, strain-rate effects are modelled at the matrix level, using a simple variation of the Weibull distribution formulation for both the modulus and the strength of RTM-6 resin [5]. Additionally, the micromechanical model enables improved

* Dimitris K. Siorikis : d.siorikis@upnet.gr 
calculation of microstresses and failure prediction at the fiber/matrix scale, and improved representation of damage effects on the updated composite properties. Finally, the incorporation of the micromechanics model reduces the amount of required material characterization testing required to model composite plies of various fiber volume ratios and laminates of various fiber architectures.

The numerical and experimental studies on the topic are further focused on carbon fiber (IMS-65) polymer (RTM-6) composites, which are extensively used in aerospace industry. A series of woven carbon/epoxy plate specimens impacted of steel ball impactors of high velocities and energies reaching and exceeding the ballistic limit $(\mathrm{m}=110 \mathrm{~g}, \mathrm{v}=60-100 \mathrm{~m} / \mathrm{s}$, $\mathrm{E}=200-500 \mathrm{~J}$ ) are investigated. and multi-scale progressive failure explicit dynamic FEA analyses are performed to assess impact response and predict progressive intraply damage and interlaminar fracture evolution. Strain-rate effects of RTM-6 are considered, according to Gerlach et al. [5] and simple variation of the Weibull distribution formulation has been adopted for both the modulus and strength of the polymer resin.. Ultimately, correlations with high-velocity impact tests and post-impact NDE validate the capability of the multi-scale FEA model to determine the structural integrity of the impacted composite structures in terms of (1) type of failure (delamination, matrix cracking, etc.), (2) damage footprint (pattern and size), (3) penetration energy (ballistic limit).

\section{Multi-scale numerical approach}

A multi-scale progressive failure framework, linking different levels of modelling refinement and enabling the efficient inclusion of strain-rate effects, was developed and implemented in Abaqus/Explicit as user material subroutine (VUMAT). The composite FE macro-mechanical response is translated to interlaminar response and fracture via cohesive zone models and to intralaminar response by the Micromechanics-VUMAT material model [4] in the representative volume element (unit cell) to assess damage initiation and damage progression in the micromechanical constituents (fibre/matrix). The degradation of the micromechanical constituent properties due to damage propagation is then translated back to degraded macro-mechanical properties. Strain-rate effects on the matrix properties are incorporated in both the failure analysis and synthesis cycles. The multi-scale progressive failure analysis process is demonstrated in Figure 1.

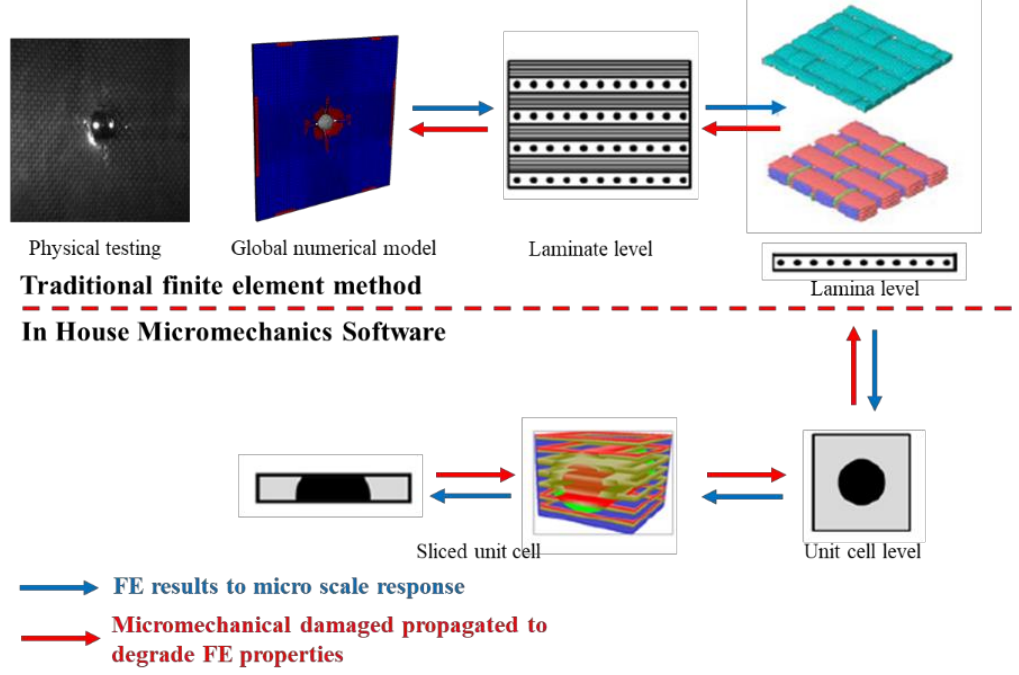

Figure 1. Graphical presentation of the multi-scale progressive failure analysis. 


\subsection{Strain-rate effects}

A simple variation of the Weibull distribution formulation was adopted to account for the rate-sensititvity of RTM-6 according to Gerlach et al. [5] and was implemented into the composite micromechanical model, which enables improved calculation of microstrains at the fiber matrix scale, thus, calculation of micro effective strain-rate of the matrix, and improved representation of rate-effects on the updated composite properties.

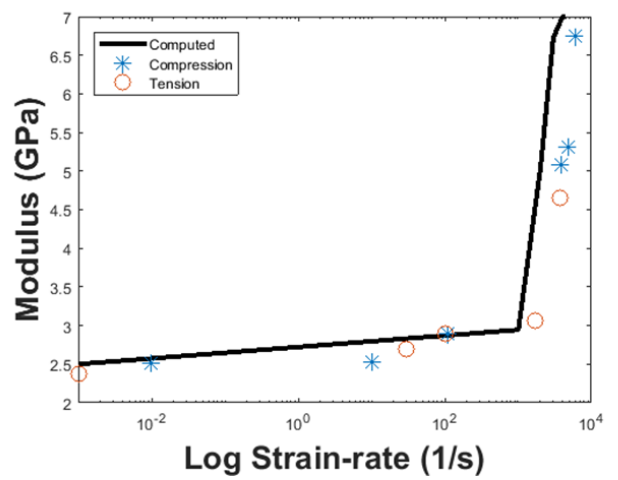

Figure 2. Modelling of strain-rate dependency of RTM-6 by using Weibull distribution.

\subsection{Building block cross-correlation approach}

A dual virtual-testing and physical-testing building block verification approach was employed to validate the predictive FEA capabilities through comparison with experimental test results as shown in Figure 10. Basic UD specimens of identical fiber/matrix system have been fabricated and tested to extract lamina properties and to back-calculate the fiber matrix properties, which provide the input of the multi-scale model. The capability of the multiscale model is evaluated on woven laminated IMS-65/RTM-6 strip specimens and plates. Finally, composite plates are impacted from steel balls and the results are correlated with multi-scale numerical prediction in terms of ballistic limit and damage footprint.

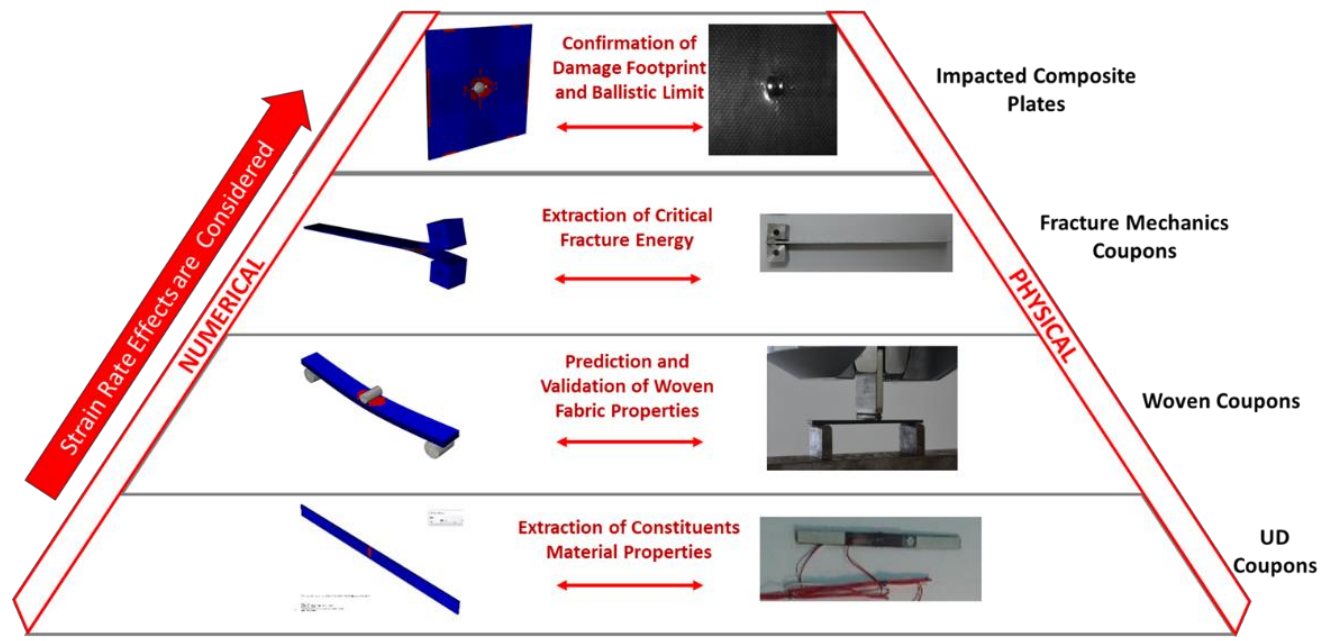

Figure 3. Dual building block approach for verification of the numerical models. 


\subsection{Material modelling verification}

Mechanical test results, in the form of raw stress, strain, and strain-rate data obtained from experimental testing (physical material characterization) were used to reverse engineers in-situ constituent mechanical properties for both the fibers and the matrix by utilizing a micromechanics-based optimization approach, as illustrated in Table 1.

Table 1. Reverse engineered (In-Situ) fiber matrix properties. (a) IMS-65. (b) RTM-6.

\begin{tabular}{lll}
\hline & IMS-65 & RTM-6 \\
\hline$E_{11}(\mathrm{GPa})$ & 288.73 & 3.32 \\
$E_{22}=E_{33}(\mathrm{GPa})$ & 3.29 & 3.32 \\
$G_{12}=G_{13}(\mathrm{GPa})$ & 93.32 & 1.12 \\
$G_{23}(\mathrm{GPa})$ & 0.92 & 1.12 \\
$v_{12}=v_{13}$ & 0.48 & 0.48 \\
$v_{23}$ & 0.48 & 0.48 \\
$S_{T}(\mathrm{GPa})$ & 3.61 & 0.09 \\
$S_{C}(\mathrm{GPa})$ & 1.40 & 0.17 \\
$S_{S}(\mathrm{GPa})$ & - & 0.45 \\
\hline
\end{tabular}

Micro-mechanics material calibration was also verified by performing multi-scale progressive failure analysis (MS-PFA) on UD specimens for classical in-plane loading conditions. Good agreement between analytical solution and test is achieved as shown in Figure 4. Moreover, a virtual three-point bending test was performed on woven specimen and the results (Figure 5) are almost identical confirming and validating the developed multiscale finite element model.

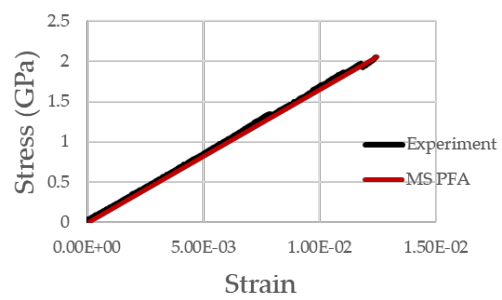

(a)

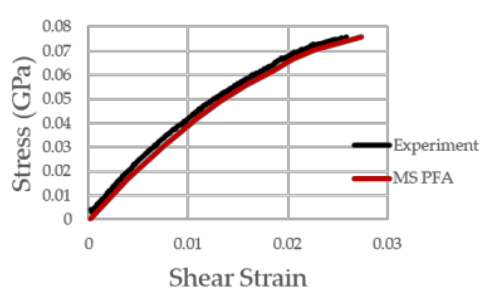

(b)

Figure 4. Material modeling calibration through multi-scale progressive failure analysis (MS-PFA) on UD specimens. (a) Longitudinal Tension. (b) Shear Tension.

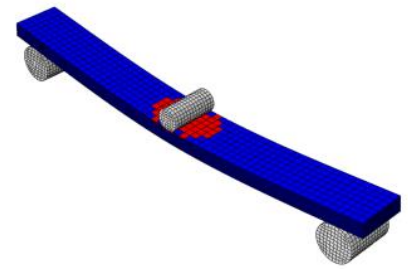

(a)

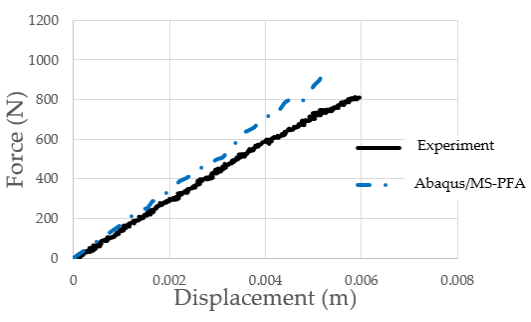

(b)

Figure 5. Multi-scale progressive failure simulation (Abaqus/MS-PFA) of three-point bending on woven specimen. (a) 3-D continuum FEA model. (b) Force-Displacement curve. 


\section{Experimental facility}

The laboratory is equipped with a functional Impact Test Bench (ITB), which mainly consists of three main parts: i) A pre-charged nitrogen gas gun. A sturdy gas gun (Figure 6) with a 10lit pressure vessel, $3 \mathrm{~m}$ barrel and $60 \mathrm{~mm}$ diameter bore is capable of launching any spherical projectile up to $50 \mathrm{~mm}$ diameter and up to $972 \mathrm{~km} / \mathrm{h}$ (velocity was tested \& verified for a $25 \mathrm{~mm}$ diameter ice ball).

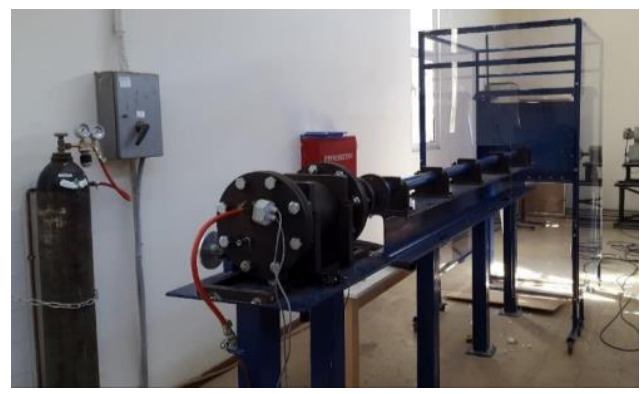

Figure 6. Impact Gas Gun

ii) Measurement devices and supporting apparatus. A high precision digital pressure gauge (Omega DPG1000DAR) for accurate measurements and repeatable impact velocities is located on the vessel. The entire impact event is captured by a high speed camera (Photron SA4) with typical 50k-100k fps capacity (Figure 7a) and two chronographs (Chrony M-1) measure both the launching and the penetration velocity at each impact case (Figure 7b).

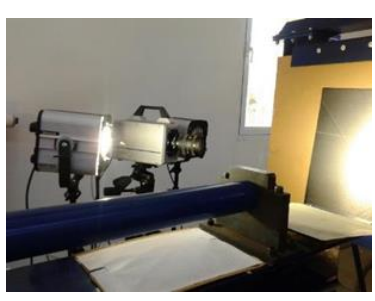

(a)

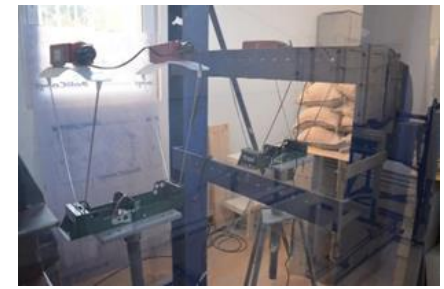

(b)

Figure 7. Measurement devices and supporting apparatus. (a) High-speed camera. (b) Chronographs for measuring launching and penetration velocity.

iii) Non-destructive testing apparatus. The lab has access to the Ultrapac II by Mistras Group (Figure $8 \mathrm{a}$ ), which provides reliable $\mathrm{C}$ scans and is used for assessing pre- and post-impact condition of the impacted structures. The post-impact condition (i.e the size of the delaminated area) is schematically validated by micromechanical models. For complex composite structures such as sandwich panels and leading edges, an ultrasonic set-up of pitchcatch is conducted using the Olympus BondMaster 1000e+(Figure 8b). 


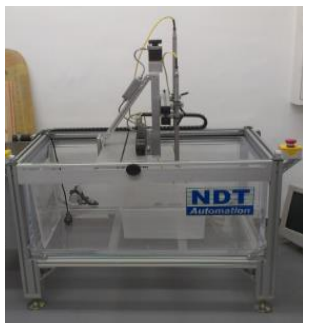

(a)

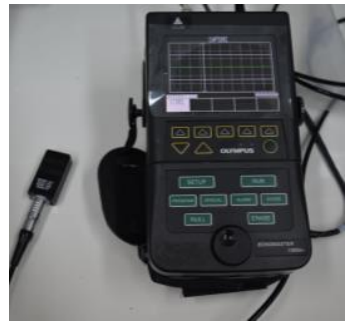

(b)

Figure 8. Non-destructive testing apparatus. (a) Ultrapac II by Mistras Group. (b) Olympus Bondmaster.

The ITB was already employed by two Cleansky projects which were efficiently closed. The gas gun has been already employed to perform an adequate number of experiments, using both hailstone and steel projectiles of various diameters, per project requirements. As for the impacted targets, flat plates, thick sandwich plates and leading edges were mounted and successfully tested.

\section{High-velocity impact results}

A series of woven IMS-65/RTM-6 plates with volume fraction $46 \%$ were fabricated using resin transfer molding (RTM) and tested under the below conditions:

1. Two impact tests with a $30 \mathrm{~mm}$ steel ball at $60 \mathrm{~m} / \mathrm{s}$ (below ballistic limit).

2. Two impact tests with a $30 \mathrm{~mm}$ steel ball at $80 \mathrm{~m} / \mathrm{s}$ (ballistic limit).

3. Two impact tests with a $30 \mathrm{~mm}$ steel ball at $100 \mathrm{~m} / \mathrm{s}$ (above ballistic limit).

In parallel, each impact case was modeled in Abaqus/Explicit through the incorporation of the multi-scale material model as user subroutine (VUMAT). The projectile was modeled using rigid shell elements (R3D4) with a diameter of $30 \mathrm{~mm}$, while the 300x300x5 mm composite plate was modeled using continuum solid elements with reduced integration (C3D8R) and geometric nonlinearity was considered to capture large displacements and rotations. All the nodes of the plate edge were fixed in all directions $(x, y, z)$ to simulate the experimental clamped conditions. The $5 \mathrm{~mm}$ thick laminate was consisted of 10 plies and was modeled using micromechanics formulation and the appropriate unit cell for woven. To ensure an adequate element size and number in the vicinity of contact region, an edge-biased technique was applied.

Damage footprint and penetration energy were compared to assess the accuracy of the proposed multi-scale finite element model, that attempts to estimate the impact resistance of the woven composite laminate. As shown in Figure 9-Figure 11, the numerical impact damage has the same shape and size with the non-destructive evaluation even in case of barely visible damage (case 1). Figure 12 shows representative snapshots from the test and the analysis, which are almost identical, ensuring the validity of the proposed model. Residual velocity (case 3) and ballistic limit were captured precisely as illustrated in Table 2. Thus, the consideration of strain-rate effects of RTM- 6 is shown to be crucial for the accurate modeling of high-velocity impact on composite structures, as illustrated by the exact predictions of damaged area and residual velocity.

Table 2. Prediction of ballistic limit and residual velocity.

\begin{tabular}{ccc}
\hline & Experiment & Simulation \\
\hline Ballistic Limit $(\mathrm{m} / \mathrm{s})$ & 80 & 75 \\
Residual Velocity $(\mathrm{m} / \mathrm{s})$ & 59.64 & 60.07 \\
\hline
\end{tabular}




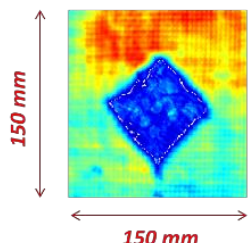

(a)

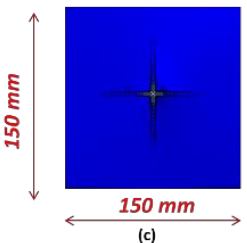

(c)

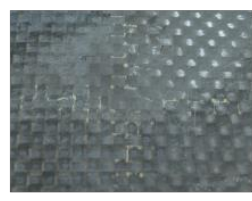

(b)

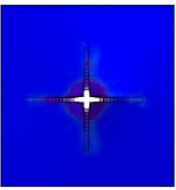

(d)

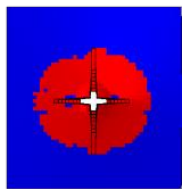

(e)

Figure 9. Correlation of damage footprint of impacted plate at $60 \mathrm{~m} / \mathrm{s}$ (case 1). (a) Post-Impact Cscan. (b) Front side of the impacted plate. (c) Fiber damage (Deleted Elements). (d) Delamination (Cohesive). (e) Matrix damage.

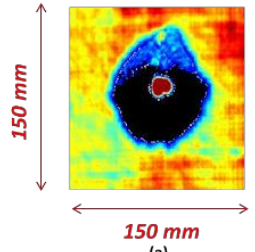

(a)

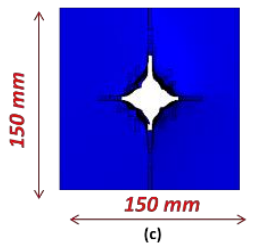

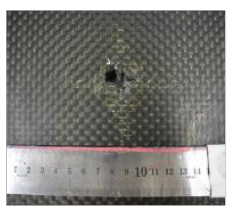

(b)

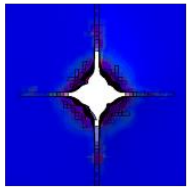

(d)

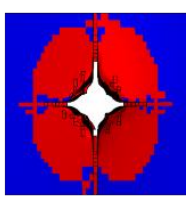

(e)

Figure 10. Correlation of damage footprint of impacted plate at $80 \mathrm{~m} / \mathrm{s}$ (case 2). (a) Post-Impact Cscan. (b) Front side of the impacted plate. (c) Fiber damage (Deleted Elements). (d) Delamination (Cohesive). (e) Matrix damage.

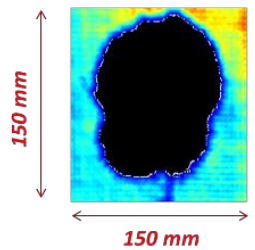

(a)

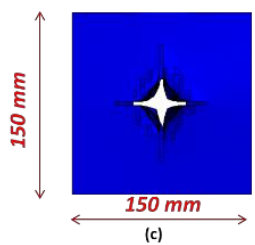

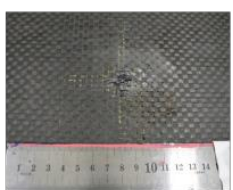

(b)

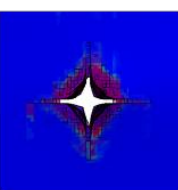

(d)

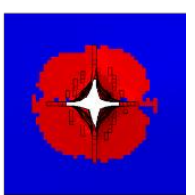

(e)

Figure 11. Correlation of damage footprint of impacted plate at $100 \mathrm{~m} / \mathrm{s}$ (case 3). (a) Post-Impact Cscan. (b) Front side of the impacted plate. (c) Fiber damage (Deleted Elements). (d) Delamination (Cohesive). (e) Matrix damage. 

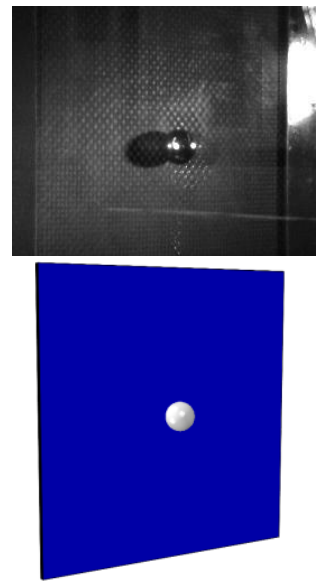

$\mathrm{t}=0 \mathrm{~s}$
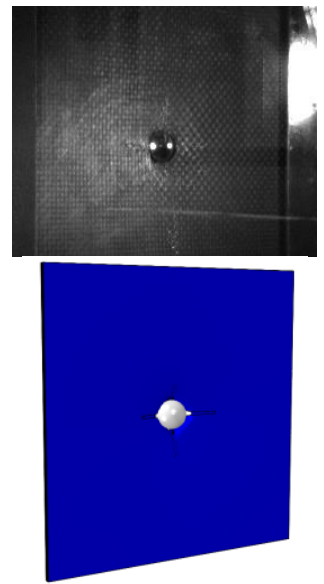

$\mathrm{t}=0.377 \mathrm{~s}$
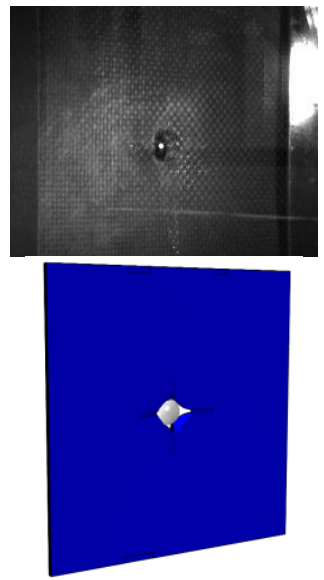

$\mathrm{t}=0.555 \mathrm{~s}$

Figure 12. Screenshots of impact test and numerical impact simulation on the woven carbon/epoxy plate $300 \times 300 \times 5 \mathrm{~mm}$ with $30 \mathrm{~mm}$ steel ball at $96 \mathrm{~m} / \mathrm{s}$.

\section{Conclusions}

A strain-rate dependent multi-scale finite element model for the simulation of high-velocity impacts on laminated composite plates has been presented, which utilizes a micromechanical composite material model with the incorporation of rate-sensitivity of RTM- 6 according to Weibull distribution. The numerical evaluations illustrated very good agreement with experimental results in terms of (1) type of failure (delamination, matrix cracking, etc.), (2) damage footprint (pattern and size), (3) penetration energy (ballistic limit). Finally, the consideration of strain-rate effects was crucial for the accurate modeling of the high velocity/rate impact event.

This work has received funding from the Clean Sky 2 Joint Undertaking under the European Union's Horizon 2020 research and innovation programme under grant agreement No [686813] - Bladeout. The authors gratefully acknowledge this support.

\section{References}

[1] Cantwell WJ, Morton J. Comparison of the low and high velocity impact response of cfrp. Composites 1989;20:545-51. doi:10.1016/0010-4361(89)90913-0.

[2] López-Puente J, Zaera R, Navarro C. Experimental and numerical analysis of normal and oblique ballistic impacts on thin carbon/epoxy woven laminates. Compos Part A Appl Sci Manuf 2008;39:374-87. doi:10.1016/j.compositesa.2007.10.004.

[3] Yen C-F. 7 th International LS-DYNA Users Conference Penetration/Explosive BALLISTIC IMPACT MODELING OF COMPOSITE MATERIALS n.d.:15-26.

[4] Chamis CC, Abdi F, Garg M, Minnetyan L, Baid H, Huang D, et al. Micromechanics-based progressive failure analysis prediction for WWFE-III composite coupon test cases. J Compos Mater 2013;47:2695-712. doi:10.1177/0021998313499478.

[5] Gerlach R, Siviour CR, Petrinic N, Wiegand J. Experimental characterisation and constitutive modelling of RTM-6 resin under impact loading. Polymer (Guildf) 2008;49:2728-37. doi:10.1016/j.polymer.2008.04.018. 